\title{
The Effects of Aerobic Exercise in Patients with Early-Onset Dementia: A Scoping Review
}

\author{
Tine Roman de Mettelinge Patrick Calders Dirk Cambier \\ Department of Rehabilitation Sciences and Physiotherapy, Faculty of Medicine and Health Sciences, Ghent \\ University, Ghent, Belgium
}

\section{Keywords}

Early-onset dementia · Exercise $\cdot$ Intervention $\cdot$ Health outcome

\begin{abstract}
Background: Early-onset dementia (EOD) defines all dementia related conditions with an onset before the age of 65 years. EOD places a large and distressing psychological, emotional and financial burden on the individuals themselves and their caregivers. For various reasons, diagnostic and treatment strategies for EOD are very challenging. There is a general agreement that not only the human body but also the mind benefits from physical activity and/or exercise. Especially aerobic exercise has shown to have favorable effects on cognitive functions in healthy older adults, as well as in patients with $\mathrm{MCl}$ and dementia. However, there are major differences in age, physical fitness level and clinical presentation between EOD and late-onset dementia. Therefore, one cannot just assume that the same type and intensity of exercise will lead to similar effects in the former population. By conducting this scoping review, the authors aimed to identify the evidence on the effectiveness of aerobic exercise on physical and mental health outcomes in individuals with EOD, display gaps in this context, and formulate related directions for future research. Summary: There are a number
\end{abstract}

of reasons to assume that aerobic exercise might be extremely valuable within individuals with EOD. However, this scoping review led to the surprising and striking finding that not a single study so far has investigated the effects of physical exercise on cognition, physical performance and feelings of well-being and quality of life in EOD. Although nowadays the disease is increasingly recognized, coping and (non-pharmacological) treatment strategies for EOD are virtually non-existent. Key Messages: Exercise intervention studies in EOD are lacking. With this scoping review the authors hope to inspire researchers in the field for related directions for future research. The potential beneficial effects of aerobic exercise in individuals with EOD should be explored and assessed extensively. Secondarily, decent guidelines for non-pharmacological treatment and coping strategies should be developed, with the aim of supporting people with EOD and their caregivers.

(c) 2021 S. Karger AG, Basel

\section{Introduction}

Early-onset dementia (EOD) defines all dementia related conditions with an onset before 65 years of age. As in late-onset dementia (LOD), prevalence rates of EOD increase with increasing age. For individuals aged 45-64
Correspondence to:

Tine Roman de Mettelinge, tine.romandemettelinge@ugent.be 
years, prevalence rate of EOD ranges from 78 to 98.1 per 100,000 [1]. EOD comprises a heterogeneous group of cognitive disorders with Alzheimer's disease (AD) as main etiology, followed by vascular dementia $(\mathrm{VaD})$ and frontotemporal dementia (FTD) $[1,2]$. Secondary dementias such as alcohol-related dementia, immunologic dementia, dementia in multiple sclerosis, traumatic brain injuries, and a large number of metabolic, infectious, neoplastic and autoimmune disorders, are more common in EOD [3]. Although AD is the most common subtype of EOD, it accounts for only $34 \%$ of EOD cases compared to $80 \%$ of LOD cases [4]. Furthermore, the clinical and imaging features of the same dementia subtypes might be different in younger individuals than older people [5]. As such, AD with early onset presents less commonly with memory deficits but tends to have a more non-amnestic presentation (worse attention, executive functions, ideomotor praxis, and visuospatial skills) than the late-onset variant [6]. Since early frontal lobe damage is common in many subtypes of EOD, EOD often presents as behavioral changes (like apathy, social withdrawal, inappropriate sexual behaviors, compulsive behavior, altered eating patterns, etc.) and personality changes (like irritability, selfishness, loss of empathy, etc.) [3]. Besides these behavioral and personality changes, other frequent early features of EOD are depression, neurological, systemic, and/ or physical symptoms (such as gait disorder, seizures, peripheral neuropathy, visual impairment). Major and/or well-known cognitive deficits may not develop until later in the disease process $[3,7]$.

Due to the wide variety of potential underlying causes (broad differential diagnosis) and the atypical clinical presentation, individuals with EOD are frequently confronted with substantial diagnostic delays and misdiagnoses. This debilitating lack of awareness might result in frustrations among the individual itself and its significant others. EOD might have divers devastating consequences as it strikes the individuals at their working age, having a prominent role in society and in providing and caring for children [8]. Therefore, EOD places a large and distressing psychologi$\mathrm{cal}$, emotional and financial burden on the individuals themselves and their caregivers, suddenly finding themselves in an unexpected and destabilized situation. Due to the interfering implications of EOD, the individual is forced to quit their job and often loses their motivation for social engagement. Participating in activities and performing household chores becomes difficult, resulting in a tremendously hampered autonomy in daily functioning.

Besides making a timely and correct diagnosis, treatment strategies and approaches for EOD are thus also very challenging. The individual with EOD has to cope with the sobering realization of their limited capabilities and find a useful way of spending their days. Given the early age of onset, individuals with EOD generally had an active lifestyle before the diagnosis was made. These intact physical abilities might, however, be threatened by the limiting cognitive capacities and related loss of initiative. Individuals with EOD often develop apathy and sedentary behaviors which may indirectly cause them to be at risk for beginning physical decline not proportional to their age. Exercise, whether or not supervised, can therefore be an excellent choice to counteract potential physical deterioration and to fill the many long hours a day suddenly counts for these individuals. Furthermore, there is a growing body of evidence that not only the human body but also the mind (cognition) benefits from physical activity and/or exercise $[9,10]$. Especially aerobic exercise has shown to have favorable effects on cognitive functions in healthy older adults [11-15], as well as in patients with MCI and dementia [16-21].

However, as described above, there are major differences in age, physical fitness level and clinical presentation between early-onset dementia and (late-onset) dementia. Therefore, one cannot just assume that the same type and intensity of exercise will lead to similar effects in the former population. By conducting this scoping review, the authors aimed to identify the evidence on the effectiveness of aerobic exercise on physical and mental health outcomes in individuals with EOD, display gaps in this context, and formulate related directions for future research.

\section{Methods}

The 5-stage framework of Arksey and O'Malley [22] was adopted to conduct this scoping review. Scoping reviews apply narrative analytic techniques and are usually not designed to assess the quality of individual studies [22, 23]. Some Preferred Reporting Items for Systematic Reviews and Meta-Analyses (PRISMA) items (such as risk of bias across studies and additional analyses) are not appropriate or applicable for a scoping review, as explained in the PRISMA extension for Scoping Reviews (PRISMAScR) by Tricco et al. [23]. These items were therefore not addressed in this scoping review. The PRISMA-ScR checklist [23] is provided in Appendix 1.

\section{Stage 1.1: Identify the Research Question}

This review is guided by the following research question: "What is known about the effects of aerobic exercise on various health outcomes in individuals diagnosed with EOD?" Three key concepts were identified from the review question: "early-onset dementia," "health outcome," and "aerobic exercise."
Roman de Mettelinge/Calders/Cambier 
Fig. 1. PRISMA flow 170 diagram for the scoping review process. PRISMA, Preferred Reporting Items for Systematic Reviews and Meta-Analyses.

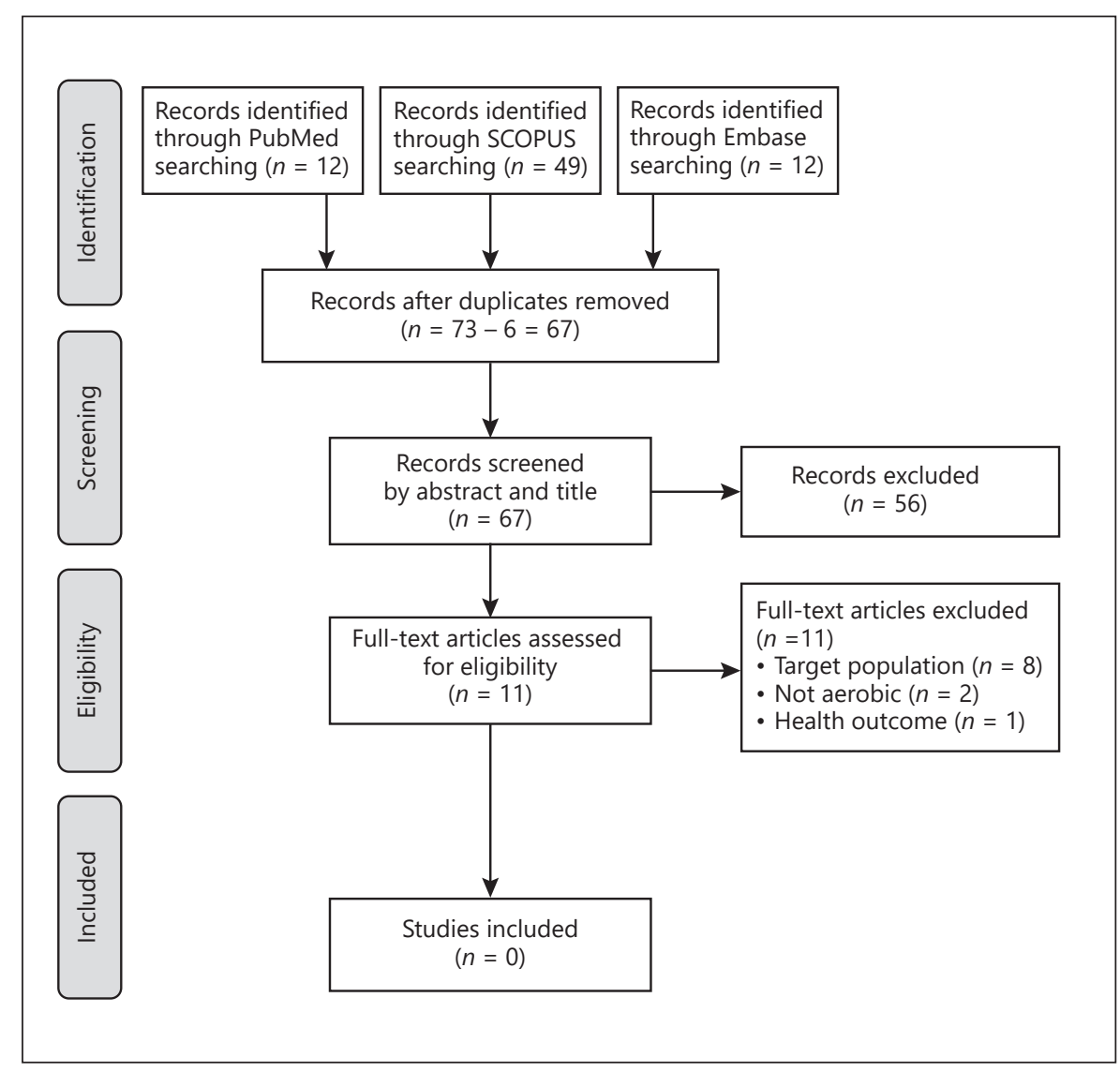

Stage 1.2: Identify the Relevant Outcomes

The potential effects of aerobic exercise in individuals with dementia were considered among three main health areas: (i) physical performance, (ii) cognitive performance, and (iii) well-being and quality of life.

Stage 2: Identifying Relevant Studies

This scoping review incorporated manuscripts that met the following inclusion criteria:

- Published in English in peer-reviewed academic journals

- Physical performance,

- Cognitive performance, and

- Well-being and quality of life.

- Designs including primary research studies (cross-sectional or longitudinal intervention designs), reviews, and meta-analyses of suitable primary research studies

- Target population: individuals with dementia, of any age, in any setting

- Publication year: no limits

Studies were excluded based on the following exclusion criteria (screening by title, abstract and/or full text):

- Walking or other exercise interventions that were not aerobic. Combined exercise interventions with an aerobic exercise component were included (e.g., brisk walking and yoga)

- Target population: other than EOD (except for healthy controls as a comparison group for people with dementia)
- Following evidence types: unpublished and/or ongoing trials, study protocols, guidelines, dissertations, conference proceedings, editorials, opinion pieces (without self-collected intervention data), case reports, articles with no primary data, other non-academic articles or health magazines

The search terms chosen for the aforementioned key concepts in PubMed were "early-onset dementia" OR "early onset dementia" OR "young-onset dementia" OR "young onset dementia" OR "presenile dementia" OR "early-onset Alzheimer" for EOD; Physical Health OR Physical Performance OR Cognition OR Cognitive Performance OR Mental Health OR Well-being OR Quality of Life OR Depression for health outcome; Aerobic Exercise OR Aerobic Training OR Physical Activity OR Physical Exercise OR Aerobic Activity OR Brisk Walking for aerobic exercise. The searches used the limiters: journal type (Peer Reviewed Journal) and language (English).

\section{Search Strategy and Databases}

Using these search terms, three electronic databases were searched during November and December 2020: PubMed, SCOPUS and Embase. The search strategy and selection process is presented as PRISMA flow diagram [24] in Figure 1.

\section{Stage 3: Study Selection}

All records identified through searching the three databases were exported to Endnote bibliographic software with abstracts and references. After the first author (T.R.d.M.) manually re- 
moved duplicates from Endnote, two researchers (T.R.d.M., P.C.) screened the remaining 67 record titles and abstracts. Abstracts with disagreement or insufficient information were screened by full texts. The first authors of 3 full text articles were contacted for access to the full text. Full-text articles with discrepancies were resolved by a third reviewer (D.C.). All three authors agreed that there were no records that met the predefined inclusion criteria.

\section{Stage 4: Charting the Data}

The intention of the authors was to extract the following key information for each included record: author(s); year; aim of the study; methodological information (design, sample size, location, and data collection); participants', controls', and intervention characteristics; and outcome measures and key findings or conclusions.

Stage 5: Collating, Summarizing, and Reporting the Results

The "descriptive-analytical" method, as described by Arksey and O'Malley [22], was meant to be applied to describe narrative summaries for each outcome. The aim was to report relevant information concerning the research question and identify research gaps.

\section{Results}

From the initial 73 records identified through searches in PubMed, SCOPUS and Embase, 11 full-text articles were retained for in-depth analysis. From these 11 fulltext articles, 8 appeared to assess a target population other than EOD, mostly (late-onset) dementia or mild cognitive impairment (MCI). The 3 remaining studies did focus on individuals with EOD but were rejected since they did not meet the inclusion criteria for type of exercise intervention and/or health outcome. Hooghiemstra et al. [25] studied the rest-activity rhythm in EOD patients. This was not a health outcome of our interest. Besides, the rest-activity rhythm was correlated with level of physical activity and not with an exercise intervention as such. The final 2 records were excluded as the type of exercise was not aerobic. Ramström incorporated a Stimulation-Activation-Training including cognitive, social and physical activities to assess the effects on QOL in early-onset AD [26], whereas Phinney et al. [27] considered the effects of leisure walks in patients with EOD in a study with a qualitative, ethnographic design. In both studies, the type of exercise or activity could not be considered a structured aerobic exercise intervention. As such, the authors remained left with zero records and could not literally implement stages 4 and 5 proposed by Arksey and O'Malley [22].

\section{Discussion}

This scoping review aimed to identify, prioritize and display gaps in the area of physical exercise among patients suffering from EOD, and to formulate related directions for future research. For the authors it was striking that not a single exercise intervention study investigating the effects on health outcomes among patients with EOD could be retained after extensive database searching. At this point, the process of conducting and writing this scoping review could have been aborted. Still, the authors opted to continue writing down their findings as they believed that the concluding message is too important not to share with researchers of interest. Although no studies could be retained for analysis and comparison of results, it is essential to report that a scoping review in this context has been conducted following the PRISMA guidelines for a scoping review process. The fact that no studies answering the predefined research question of this review could be found, is a surprising but also a critical finding as it proves that scientific research focusing on the effects of exercise interventions on health outcomes in EOD is nonexistent.

As stated in the introduction, there is a general agreement that not only the human body but also the mind (cognition) benefits from physical activity and/or exercise $[9,10]$. Especially, aerobic exercise has shown to have favorable effects on cognitive functions in healthy older adults [11-15], as well as in patients with MCI and dementia [16-21], with the largest gains demonstrated in executive functioning $[9-12,14,15,28]$. Other studies have reported the greatest benefits on cognition, physical functions, mood and behavior after multicomponent exercise interventions (e.g., combined strength and aerobic exercises) in patients with MCI [28] and dementia [28-30]. From their interesting umbrella review, Demurtas et al. [28] concluded that mixed physical activity interventions had a small effect on global cognition in people with MCI and a medium to large effect on global cognition in people with dementia. Second, mind-body interventions (e.g., tai chi and yoga) had a small effect on global cognition, attention and executive function in people with MCI and a medium effect on memory [28]. Third, resistance training interventions had a large effect on global cognition in people with MCI [28]. Besides the beneficial effects on divers cognitive domains, they also indicated that physical activity/exercise significantly decreased risk and number of falls, and that physical activity/exercise may improve depression and behavioral and psychological symptoms in dementia 
[28]. Finally, some recent randomized controlled trials have shown that a combined exercise and cognitive training improves attention, concentration, cognitive function and activities of daily living in dementia patients [31] and is effective in delaying/preventing cognitive deterioration and cognitive functional decline in people with MCI [32]. Although there is no consensus on the most effective modalities, it is remarkable that exercise (single- or multicomponent) seems to be a constant key factor in this context. Still, all researchers in the field agree that more high-quality studies are needed to elucidate the effects and underlying mechanisms of different and/or combined exercise types on cognition in brain disorders [28,29]. Future research should focus on developing specific exercise programs and guidelines to reduce the risk or slow down disease progression of dementia $[33,34]$. Nevertheless, there is clearly considerable evidence to assume that exercise might and should play a fundamental role in coping and treatment strategies for further cognitive decline, even in older adults with structural brain damage such as MCI or (late-onset) dementia. However, it remains remarkably quiet as it comes to exercise intervention studies among younger individuals with EOD.

Still, there is some growing awareness for the need of non-pharmacological intervention studies in EOD. As such, a protocol for a scoping review entitled "Physical activity for people with young-onset dementia and carers" was developed in 2018 by Rodgers et al. [35]. Six years earlier, Hooghiemstra et al. [36] launched theoretical considerations concerning the value of exercise interventions in early-onset AD. From their review they concluded that brain regions and neurobiological processes contributing to the positive effects of exercise are affected in early-onset $\mathrm{AD}$, thereby supporting exercise interventions in this population [36]. The same research group established a decent study protocol aiming to evaluate whether exercise slows down the progressive course of the symptoms of dementia in EOD patients [37]. Nevertheless, no results of this or another exercise intervention study in EOD has been published so far. This is a surprising finding as there are a number of reasons to assume that exercise might be extremely valuable within this population. First, as mentioned above, the positive effects of exercise on cognition have been proven earlier in healthy individuals and LOD. Therefore, it is most likely that individuals with EOD will also benefit from exercise interventions. It might even be hypothesized that physical exercise not only slows down the progression of cognitive decline in EOD but also has favorable effects on behavior, feelings of well-being and quality of life (for the individual itself and their supportive network). Second, the impact of exercise may even be greater in EOD compared to LOD as it is generally recognized that the intensity and/or frequency of physical exercise is highly responsible for its outcome. Given their relatively young age, active lifestyle and generally better understanding of instructions, individuals with EOD are more eligible to participate in more intensive and/or complex training programs compared to individuals with LOD with age-related comorbidities. However, results from previous research suggest that the threshold of intensity that reduces the risk of cognitive decline and dementia is probably low [33]. Third, the non-pharmacological approach of individuals with EOD as well as formal and informal support for the caregivers is very limited. Engaging the individual with EOD in activities such as exercise might be an excellent choice of distraction and an important component of coping strategies.

Despite the undeniable beneficial consequences, not a single study so far has investigated the effects of physical exercise on cognition, physical performance and feelings of well-being and quality of life (QOL) in EOD. How comes that this trainable population, seeking for nonpharmacological treatment and coping strategies, is neglected as it comes to scientific research in this area? As mentioned earlier, EOD covers a rather small proportion of all dementia conditions and can be considered a rare condition. Generally, people with EOD live in residential aged care facilities (primarily developed for older individuals) or at home, relying on their supporting family and optionally day-care services. The intrinsic motivation of people with EOD might be low as a direct consequence of the disease. Caregivers often live a very stressful life, combining their job with the care and maintenance of their family. Therefore, it is expected that it is not only hard to track these people but also to convince them to participate in intense and long-term exercise intervention studies. These considerable recruitment challenges might explain why conclusive evidence concerning the effect of exercise in individuals with EOD is lacking. Still, feasible initiatives to deal with this motivational challenge are thinkable. Brisk walking has proven to be a feasible, safe, and effective form of moderate-intensity aerobic physical exercise [38-40] that is assumed to be healthenhancing for the individuals with EOD and their accompanying caregivers. Besides, as stated above, a solid study protocol has been proposed earlier by Hooghiemstra et al. [37]. 


\section{Conclusions}

The answer on the research question "What is known about the effects of aerobic exercise on various health outcomes (i.e., physical performance, cognitive performance, and well-being/QOL) in individuals diagnosed with EOD?" is very straightforward: Nothing. Not a single study seemed to be suitable for inclusion in this scoping review. As such, this scoping review identified a huge research gap. Although nowadays the disease is increasingly recognized, coping and (non-pharmacological) treatment strategies for EOD are virtually non-existent. To the authors' opinion, this is the first review that systematically explored the current state of evidence regarding the effects of aerobic exercise among people with EOD. With this scoping review the authors hope to inspire researchers in the field for related directions for future research. Although promising research has been conducted in people with related cognitive disorders such as MCI and dementia, the potential beneficial effects of aerobic exercise should be explored and assessed extensively among individuals with EOD. Secondarily, decent guidelines for non-pharmacological treatment and coping strategies should be developed, with the aim of supporting people with EOD and their caregivers.

\section{Statement of Ethics}

The 5-stage framework of Arksey and O'Malley [22] was adopted to conduct this scoping review. Scoping reviews apply narrative analytic techniques and are usually not designed to assess the quality of individual studies [22, 23]. Some Preferred Reporting Items for Systematic Reviews and Meta-Analyses (PRISMA) items (such as risk of bias across studies and additional analyses) are not appropriate or applicable for a scoping review, as explained in the PRISMA extension for Scoping Reviews (PRISMA-ScR) by Tricco et al. [23]. These items were therefore not addressed in this scoping review. The PRISMA-ScR checklist [23] is provided in the Appendix 1.

\section{Conflict of Interest Statement}

The authors have no conflicts of interest to declare.

\section{Funding Sources}

This scoping review received no specific grant from any funding agency in the public, commercial, or not-for-profit sectors.

\section{Author Contributions}

T.R.d.M. conceived the scoping review. T.R.d.M., P.C., and D.C. contributed to screening records. T.R.d.M. drafted the full manuscript. P.C. and D.C. profoundly reviewed the manuscript and approved final submission.

\section{Appendix 1}

\begin{tabular}{|c|c|c|c|}
\hline Section & No. & PRISMA-ScR checklist item & $\begin{array}{l}\text { Reported } \\
\text { on page }\end{array}$ \\
\hline \multicolumn{4}{|l|}{ Title } \\
\hline Title & 1 & Identify the report as a scoping review & 1 \\
\hline \multicolumn{4}{|l|}{ Abstract } \\
\hline Structured summary & 2 & $\begin{array}{l}\text { Provide a structured summary that includes (as applicable) background, objectives, eligibility } \\
\text { criteria, sources of evidence, charting methods, results, and conclusions that relate to the review } \\
\text { questions and objectives }\end{array}$ & 2 \\
\hline \multicolumn{4}{|l|}{ Introduction } \\
\hline Rationale & 3 & $\begin{array}{l}\text { Describe the rationale for the review in the context of what is already known. Explain why the } \\
\text { review questions/objectives lend themselves to a scoping review approach }\end{array}$ & 3,4 \\
\hline Objectives & 4 & $\begin{array}{l}\text { Provide an explicit statement of the questions and objectives being addressed with reference to } \\
\text { their key elements (e.g., population or participants, concepts, and context) or other relevant key } \\
\text { elements used to conceptualize the review questions and/or objectives }\end{array}$ & 4 \\
\hline \multicolumn{4}{|l|}{ Methods } \\
\hline Protocol and registration & 5 & $\begin{array}{l}\text { Indicate whether a review protocol exists; state if and where it can be accessed (e.g., a Web } \\
\text { address); and if available, provide registration information, including the registration number }\end{array}$ & 4,5 \\
\hline
\end{tabular}




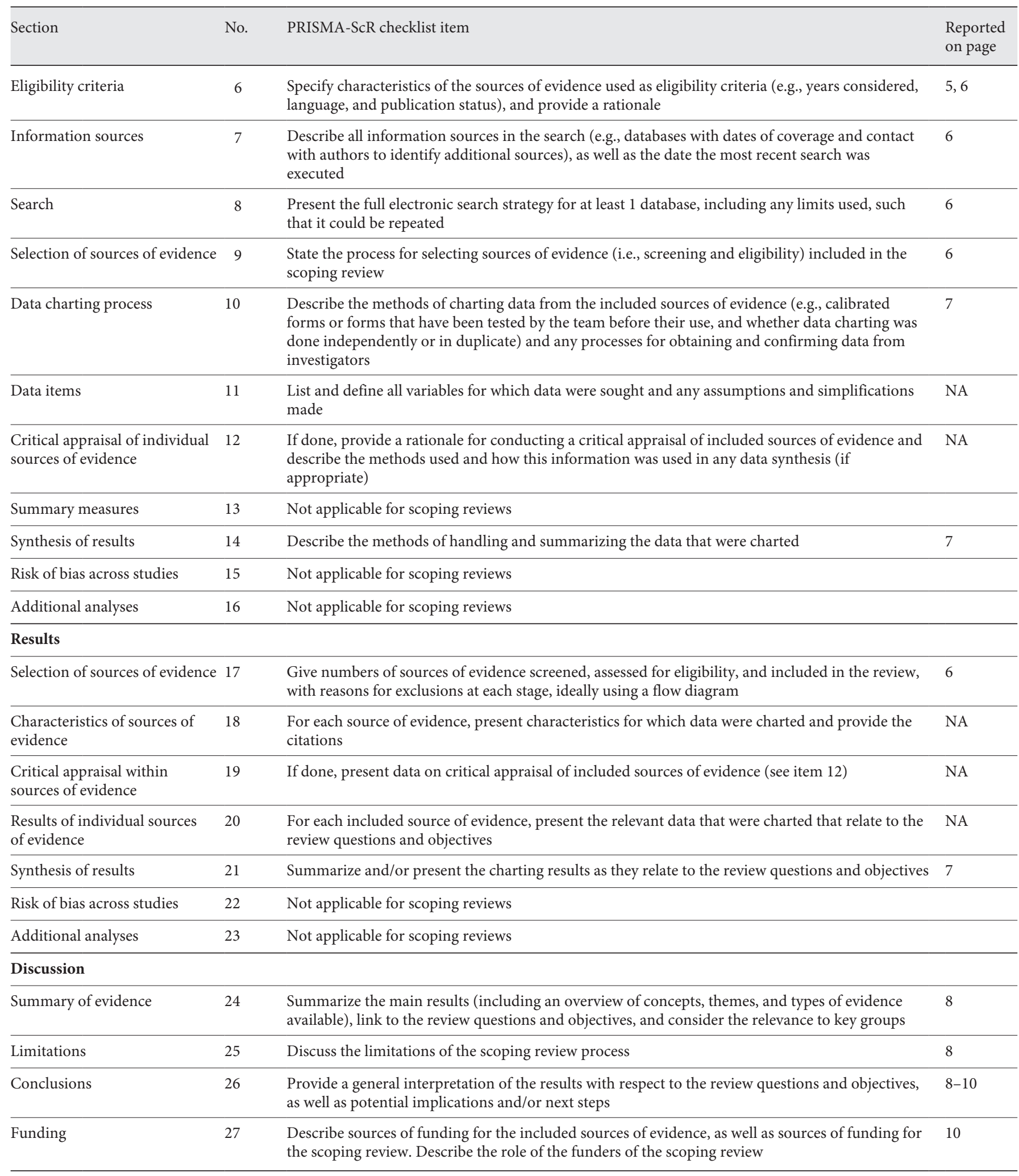

PRISMA-ScR Checklist [23]. 


\section{References}

1 Vieira RT, Caixeta L, Machado S, Silva AC, Nardi AE, Arias-Carrión O, et al. Epidemiology of early-onset dementia: a review of the literature. Clin Pract Epidemiol Ment Health. 2013;9:88-95.

2 Rossor MN, Fox NC, Mummery CJ, Schott JM, Warren JD. The diagnosis of young-onset dementia. Lancet Neurol. 2010;9(8):793-806.

3 Draper B, Withall A. Young onset dementia. Intern Med J. 2016;46(7):779-86.

4 Harvey RJ, Skelton-Robinson M, Rossor MN. The prevalence and causes of dementia in people under the age of 65 years. J Neurol Neurosurg Psychiatry. 2003;74(9):1206-9.

5 Quach C, Hommet C, Mondon K, Lauvin MA, Cazals X, Cottier JP. Early-onset dementias: specific etiologies and contribution of MRI. Diagn Interv Imaging. 2014;95(4):37798.

6 Mendez MF. Early-onset Alzheimer disease. Neurol Clin. 2017;35(2):263-81.

7 Kuruppu DK, Matthews BR. Young-onset dementia. Semin Neurol. 2013;33(4):365-85.

8 McMurtray A, Clark DG, Christine D, Mendez MF. Early-onset dementia: frequency and causes compared to late-onset dementia. Dement Geriatr Cogn Disord. 2006;21(2):59-64.

9 Smith PJ, Blumenthal JA, Hoffman BM, Cooper H, Strauman TA, Welsh-Bohmer K, et al. Aerobic exercise and neurocognitive performance: a meta-analytic review of randomized controlled trials. Psychosom Med. 2010; 72(3):239-52

10 Voss MW, Nagamatsu LS, Liu-Ambrose T, Kramer AF. Exercise, brain, and cognition across the life span. J Appl Physiol. 2011; 111(5):1505-13.

11 Angevaren M, Aufdemkampe G, Verhaar HJ Aleman A, Vanhees L. Physical activity and enhanced fitness to improve cognitive function in older people without known cognitive impairment. Cochrane Database Syst Rev. 2008;3:CD005381.

12 Colcombe S, Kramer AF. Fitness effects on the cognitive function of older adults: a meta-analytic study. Psychol Sci. 2003;14(2):125-30.

13 Erickson KI, Voss MW, Prakash RS, Basak C, Szabo A, Chaddock L, et al. Exercise training increases size of hippocampus and improves memory. Proc Natl Acad Sci U S A. 2011; 108(7):3017-22.

14 Rosano C, Venkatraman VK, Guralnik J, Newman AB, Glynn NW, Launer L, et al. Psychomotor speed and functional brain MRI 2 years after completing a physical activity treatment. J Gerontol A Biol Sci Med Sci. 2010;65(6):639-47.

15 Voss MW, Prakash RS, Erickson KI, Basak C, Chaddock L, Kim JS, et al. Plasticity of brain networks in a randomized intervention trial of exercise training in older adults. Front Aging Neurosci. 2010;2:32.
16 Groot C, Hooghiemstra AM, Raijmakers PG, van Berckel BN, Scheltens P, Scherder EJ, et al. The effect of physical activity on cognitive function in patients with dementia: a metaanalysis of randomized control trials. Ageing Res Rev. 2016;25:13-23.

17 Tekeşin A, Tunç A, Güngen BD, Avcı N, Bakış $\mathrm{M}$, Perk S. Pulmonary physiotherapy and aerobic exercise programs can improve cognitive functions and functional ability. Ideggyogy Sz. 2018;71(11-12):423-30.

18 Lange-Asschenfeldt C, Kojda G. Alzheimer's disease, cerebrovascular dysfunction and the benefits of exercise: from vessels to neurons. Exp Gerontol. 2008;43(6):499-504.

19 Öhman H, Savikko N, Strandberg TE, Pitkälä $\mathrm{KH}$. Effect of physical exercise on cognitive performance in older adults with mild cognitive impairment or dementia: a systematic review. Dement Geriatr Cogn Disord. 2014; 38(5-6):347-65.

20 Li Z, Chen Q, Liu J, Du Y. Physical exercise ameliorates the cognitive function and attenuates the neuroinflammation of Alzheimer's disease via miR-129-5p. Dement Geriatr Cogn Disord. 2020;49(2):163-9.

21 Kemoun G, Thibaud M, Roumagne N, Carette P, Albinet C, Toussaint L, et al. Effects of a physical training programme on cognitive function and walking efficiency in elderly persons with dementia. Dement Geriatr Cogn Disord. 2010;29(2):109-14.

22 Arksey H, O’Malley L. Scoping studies: towards a methodological framework. Int J Soc Res Methodol. 2005;8(1):19-32.

23 Tricco AC, Lillie E, Zarin W, O’Brien KK, Colquhoun H, Levac D, et al. PRISMA extension for scoping reviews (PRISMA-ScR): checklist and explanation. Ann Intern Med. 2018;169(7):467-73.

24 Moher D, Liberati A, Tetzlaff J, Altman DG. Preferred reporting items for systematic reviews and meta-analyses: the PRISMA statement. BMJ. 2009;339(7):b2535.

25 Hooghiemstra AM, Eggermont LH, Scheltens $\mathrm{P}$, van der Flier WM, Scherder EJ. The restactivity rhythm and physical activity in earlyonset dementia. Alzheimer Dis Assoc Disord. 2015;29(1):45-9.

26 Ramström I. Stimulation-activation-training in early-onset Alzheimer's disease: a pilot study. J Chin Clin Med. 2010;5(7):425-32.

27 Phinney A, Kelson E, Baumbusch J, O’Connor $\mathrm{D}$, Purves B. Walking in the neighbourhood: performing social citizenship in dementia. Dementia. 2016;15(3):381-94.

28 Demurtas J, Schoene D, Torbahn G, Marengoni A, Grande G, Zou L, et al. Physical activity and exercise in mild cognitive impairment and dementia: an umbrella review of intervention and observational studies. J Am Med Dir Assoc. 2020;21(10):1415-22.e6.
29 Henskens M, Nauta IM, Van Eekeren MCA, Scherder EJA. Effects of physical activity in nursing home residents with dementia: a randomized controlled trial. Dement Geriatr Cogn Disord. 2018;46(1-2):60-80.

30 Blankevoort CG, van Heuvelen MJ, Boersma F, Luning H, de Jong J, Scherder EJ. Review of effects of physical activity on strength, balance, mobility and ADL performance in elderly subjects with dementia. Dement Geriatr Cogn Disord. 2010;30(5):392-402.

31 Okamura H, Otani M, Shimoyama N, Fujii T. Combined exercise and cognitive training system for dementia patients: a randomized controlled trial. Dement Geriatr Cogn Disord. 2018;45(5-6):318-25.

32 Griffiths J, Thaikruea L, Wongpakaran N, Munkhetvit P, Kittisares A, Varnado P. Effects of combined physical movement activity and multifaceted cognitive training in older people with mild neurocognitive disorder in a rural community: a randomized control trial. Dement Geriatr Cogn Disord. 2020;49(2):194-201.

33 Rolland Y, Abellan van Kan G, Vellas B. Physical activity and Alzheimer's disease: from prevention to therapeutic perspectives. J Am Med Dir Assoc. 2008;9(6):390-405.

34 Kelly P, Williamson C, Niven AG, Hunter R, Mutrie N, Richards J. Walking on sunshine: scoping review of the evidence for walking and mental health. Br J Sports Med. 2018; 52(12):800-6.

35 Rodgers C, Rogerson D, Stevenson J, Porock D. Physical activity for people with youngonset dementia and carers: protocol for a scoping review. Syst Rev. 2018;7(1):36.

36 Hooghiemstra AM, Eggermont LH, Scheltens $\mathrm{P}$, van der Flier WM, Scherder EJ. Exercise and early-onset Alzheimer's disease: theoretical considerations. Dement Geriatr Cogn Dis Extra. 2012;2(1):132-45.

37 Hooghiemstra AM, Eggermont LH, Scheltens $\mathrm{P}$, van der Flier WM, Bakker J, de Greef MH, et al. Study protocol: EXERcise and cognition in sedentary adults with early-ONset dementia (EXERCISE-ON). BMC Neurol. 2012;12:75.

38 Kukkonen-Harjula K, Hiilloskorpi H, Mänttäri A, Pasanen M, Parkkari J, Suni J, et al. Self-guided brisk walking training with or without poles: a randomized-controlled trial in middle-aged women. Scand J Med Sci Sports. 2007;17(4):316-23.

39 Lee IM, Buchner DM. The importance of walking to public health. Med Sci Sports Exerc. 2008;40(7 Suppl):S512-8.

40 Haskell WL, Lee IM, Pate RR, Powell KE, Blair SN, Franklin BA, et al. Physical activity and public health: updated recommendation for adults from the American College of Sports Medicine and the American Heart Association. Med Sci Sports Exerc. 2007;39(8): 1423-34. 